\title{
Definition of Clinically Meaningful Within-Patient Changes in POEM and CDLQI in Children 6 to 11 Years of Age with Severe Atopic Dermatitis
}

\author{
Eric L. Simpson (D) - Marjolein de Bruin-Weller • Ashish Bansal • \\ Zhen Chen · Lauren Nelson · Diane Whalley • Randy Prescilla • \\ Isabelle Guillemin • Dimittri Delevry
}

Received: March 1, 2021 / Published online: May 27, 2021

(C) The Author(s) 2021

\begin{abstract}
Introduction: The Patient-Oriented Eczema Measure (POEM) assesses patient-reported severity of atopic dermatitis (AD) symptoms, whereas the Children's Dermatology Life Quality Index (CDLQI) measures how $\mathrm{AD}$ affects health-related quality of life (HRQoL) in children. Although the POEM and CDLQI have established thresholds for clinically meaningful
\end{abstract}

Supplementary Information The online version contains supplementary material available at https:// doi.org/10.1007/s13555-021-00543-7.

E. L. Simpson ( $₫)$

Department of Dermatology, Oregon Health and Science University, Portland, OR, USA

e-mail: simpsone@ohsu.edu

M. de Bruin-Weller

University Medical Center Utrecht (UMCU),

Utrecht, The Netherlands

A. Bansal · Z. Chen · D. Delevry

Regeneron Pharmaceuticals, Inc, Tarrytown, NY, USA

L. Nelson

RTI Health Solutions, Durham, NC, USA

D. Whalley

RTI Health Solutions, Didsbury, Manchester, UK

R. Prescilla

Sanofi Genzyme, Cambridge, MA, USA

I. Guillemin

Sanofi, Lyon, France within-patient change in adolescents (aged 12-17 years), there are no defined within-patient responder thresholds for clinically meaningful change in children aged 6-11 years.

Methods: Data from the LIBERTY AD PEDS phase 3 randomized, double-blind, placebocontrolled trial of dupilumab in children aged 6-11 years with severe $A D$ were used to define the threshold for within-patient meaningful change in POEM and CDLQI scores. Anchorbased methods were applied to estimate mean change in POEM and CDLQI scores from baseline to week 16, with anchors of a 1-point improvement in the Patient Global Impression of Disease (PGID) scale and an improvement in score of "A little better" on the Patient Global Impression of Change (PGIC) scale. The distribution-based methods, a one-half standard deviation (SD) at baseline and a standard error mean (SEM) were also used.

Results: The mean POEM change scores associated with the anchors were a change of -8.40 with the PGID anchor and -6.30 with the PGIC anchor. Distribution-based estimates for POEM were one-half SD at baseline of 2.76, with a SEM of 3.32. Mean CDLQI change scores corresponding to the PGID and PGIC anchors were -7.30 and -6.80 , respectively, while distribution-based estimates for CDLQI were a one-half SD at baseline of 3.69, with a SEM of 3.52.

Conclusions: In children with severe $\mathrm{AD}$, an appropriate minimum threshold of clinically meaningful within-patient change was 
estimated as 6 points for both the POEM and CDLQI scores.

Trial registration: ClinicalTrials.gov Identifier: NCT03345914.

Keywords: Atopic dermatitis; CDLQI; Clinically meaningful; Dupilumab; Pediatric; POEM; Psychometric; Responder threshold; Validation

\section{Key Summary Points}

\section{Why carry out this study?}

The Patient-Oriented Eczema Measure (POEM) and Children's Dermatology Life Quality Index (CDLQI) questionnaires are widely used but do not have established thresholds for clinically meaningful within-patient change for children aged 6-11 years.

Data from the phase 3 study LIBERTY AD PEDS were used to estimate thresholds of meaningful change in children with severe AD using anchor-based and distribution-based methods.

\section{What was learned from the study?}

For children aged 6-11 years with severe $\mathrm{AD}$, an appropriate threshold of clinically meaningful within-patient change was estimated as 6 points for both the POEM and the CDLQI scores.

The data presented herein generally agree with and build upon previous publications that estimated clinically meaningful within-patient change thresholds for POEM and CDLQI scores in patients with similar clinical phenotypes.

\section{DIGITAL FEATURES}

This article is published with digital features, including a summary slide and video summary, to facilitate understanding of the article. To view digital features for this article go to https:// doi.org/10.6084/m9.figshare.14484600.

\section{INTRODUCTION}

Atopic dermatitis (AD) is a chronic, lifelong disease with a high patient burden that is under-recognized as a public health concern [1-3]. It is the most common childhood inflammatory skin disease, affecting more than $20 \%$ of children in many industrialized countries, with a continually increasing prevalence [4-6].

The Patient-Oriented Eczema Measure (POEM) was developed as a tool for assessing patient-reported severity of AD in clinical practice and clinical trials in adults and children [7]. The instrument has been recommended by Harmonising Outcome Measures for Eczema (HOME), an international initiative formed to establish a set of core outcomes for AD trials, as a core outcome instrument to assess symptom severity in the AD population [8]. The Children's Dermatology Life Quality Index (CDLQI) was developed to measure dermatology-specific health-related quality of life (HRQoL) in children [9]. CDLQI is well established and widely used; from 1995 to 2012, the measure was used in 102 studies across 14 different skin conditions [10]. Both instruments have been validated in the pediatric population, showing good validity and reliability [7, 9, 11-23].

A threshold of 6-8 points for clinically relevant within-patient response or responder definition has been established in adolescents with $\mathrm{AD}$ for both the POEM and CDLQI [24] scores. For adults with $\mathrm{AD}$, a responder threshold for clinically meaningful change of 4 points has been established for both POEM [25] and Dermatology Life Quality Index (DLQI) [26]. However, there is currently no validated responder threshold for clinically meaningful change in these instruments for children with $\mathrm{AD}$ (aged 6-11 years). This article describes the empirical derivation of within-patient thresholds of clinically meaningful change in POEM and CDLQI scores in children with severe $\mathrm{AD}$. 


\section{METHODS}

This analysis is based on data from a randomized, double-blind, placebo-controlled trial of dupilumab in children with severe AD. Detailed descriptions of the study population and methodology are reported elsewhere [27] and are briefly summarized here.

The study was conducted in accordance with the provisions of the Declaration of Helsinki, the International Council for Harmonisation Good Clinical Practice (ICH GCP) guideline, and applicable regulatory requirements; the protocol was reviewed and approved by institutional review boards/ethics committees. For all patients, written informed consent was obtained from a parent or legal guardian and written informed assent was obtained from the patient.

\section{Study Design}

The LIBERTY AD PEDS (NCT03345914) phase 3 trial included children (6-11 years of age) with severe $\mathrm{AD}$ inadequately controlled with topical medications or for whom topical treatment is medically inadvisable [27]. Patients were randomized in a 1:1:1 ratio to dupilumab administered concomitantly with topical corticosteroids every 2 weeks ( $100 \mathrm{mg}$ for children $<30 \mathrm{~kg}$ or $200 \mathrm{mg}$ for children $\geq 30 \mathrm{~kg}$ ) or every 4 weeks (300 mg), or placebo for 16 weeks. For the purpose of this analysis all patients were included irrespective of treatment arm.

Patient demographics and background have been reported in detail previously [27]. Briefly, among the 367 patients randomized in the LIBERTY AD PEDS trial, the average age (standard deviation [SD] ) was 8.5 (1.72) years, $49.9 \%$ were male, $69.2 \%$ were White, $16.9 \%$ were Black/African American, $7.6 \%$ were Asian, and $5.2 \%$ were of a race that did not fall into the previous categories.

\section{Patient-Reported Outcome Measures}

The POEM comprises seven items that assess the frequency of $\mathrm{AD}$ symptoms (itchy, bleeding, weeping or oozing, cracked, flaky, and dry or rough skin) and sleep disturbance over the past week [7]. Items are rated on a 5-point scale ranging from no days (scored 0 ) to every day (scored 4). The total score ranges from 0 to 28 , with higher scores indicating greater frequency of $\mathrm{AD}$ symptoms and of sleep disturbance. POEM was completed by caregivers during clinic visits at screening, baseline (week 0), and weeks $2,4,8,12$, and 16 (end of treatment).

The CDLQI comprises ten items assessing the impact of skin disease on children's HRQoL over the previous week [9]. The items cover symptoms, leisure activities, school or holiday time, personal relationships, sleep, side effects of treatment, and emotional reactions to having a skin disease. The total score ranges from 0 to 30 , with higher scores indicating greater impairment in HRQoL. CDLQI was completed by children at clinic visits at screening, baseline (week 0), and weeks 2, 4, 8, 12, and 16 (end of treatment).

\section{Estimation of Within-Patient Change}

Anchor-based and distribution-based methods were applied to estimate the threshold for meaningful within-patient change in POEM and CDLQI scores. For the anchor-based approach, the mean change scores for POEM and CDLQI were calculated based on defined improvement in the selected anchors. In line with recommendations that patient-reported anchors (often global assessments) are the most appropriate [28], a 1-point improvement on the Patient Global Impression of Disease (PGID) scale and "a little better" on the Patient Global Impression of Change (PGIC) scale from baseline to week 16 were selected as the anchors. The PGID questionnaire asked participants about their itching in the last 7 days and was scored on a 5-point scale (not itchy at all, a little itchy, medium itchy, pretty itchy, very itchy). The PGIC questionnaire is designed to measure the perceived change in itching since starting medication and is scored on a 5-point scale (much better, a little better, the same, a little worse, much worse). Distribution-based methods for determining responder thresholds were considered as a supportive approach based on 
guidance from the US Food and Drug Administration [29]. The distribution-based methods included one-half SD at baseline and standard error of measurement (SEM) computed as SD $\sqrt{1-r}$, where $r$ is the test-retest intraclass correlation coefficient.

\section{RESULTS}

Of the 367 patients randomized in the LIBERTY AD PEDS trial, 357 and 356 caregivers/patients, respectively, completed baseline and week 16 POEM and CDLQI assessments and received at least one dose of dupilumab or placebo.

Both the PGID and PGIC were confirmed to be appropriate anchors, based on the magnitude of change correlations for POEM $(|r|=0.65$ with PGID and 0.60 with PGIC) and CDLQI $(|r|$ $=0.58$ with PGID and 0.44 with PGIC), which were above the minimum recommended correlation of 0.37 for anchor measures [30-32].

The mean POEM change scores associated with a PGID improvement of 1 point and a PGIC score of "a little better" improvement were -8.40 and -6.30 , respectively (Table 1 ). The distribution-based estimates were a onehalf SD at baseline of 2.76 and a SEM of 3.32. The mean CDLQI change scores associated with the anchors were -7.30 with PGID and -6.80 with PGIC (Table 2). The distribution-based one-half SD was 3.69 at baseline and the SEM was 3.52 .

\section{DISCUSSION}

In the absence of thresholds to interpret meaningful within-patient change in POEM and CDLQI scores specifically for children aged 6-11 years with $A D$, data from the phase 3 LIBERTY AD PEDS were used to define thresholds in POEM and CDLQI scores for the current target population, using anchor-based and distribution-based methods. In a recent paper, Simpson et al. [24] established a within-patient change of $6-8$ points in POEM and CDLQI scores as a reasonable responder threshold for clinically meaningful change in each of the two
Table 1 Estimates for thresholds for meaningful withinpatient change on the Patient-Oriented Eczema Measure using data from the phase 3 randomized, double-blind, placebo-controlled LIBERTY AD PEDS trial

\begin{tabular}{|c|c|c|}
\hline \multirow[t]{2}{*}{ Method } & \multicolumn{2}{|c|}{$\begin{array}{l}\text { Change in } \\
\text { POEM score } \\
\text { from baseline to } \\
\text { week } 16^{\mathrm{a}} \\
(N=357)\end{array}$} \\
\hline & Mean & Median \\
\hline \multicolumn{3}{|l|}{ Anchor based } \\
\hline $\begin{array}{l}\text { PGID improvement of } 1 \text { point } \\
(n=93)\end{array}$ & -8.40 & -9.00 \\
\hline $\begin{array}{l}\text { PGIC improvement "a little better" } \\
(n=84)\end{array}$ & -6.30 & -5.00 \\
\hline
\end{tabular}

Distribution based

One-half SD at baseline $\quad 2.76$

$\mathrm{SEM}^{\mathrm{b}} \quad 3.32$

The analysis sample included 357 randomized patients who received at least one dose of dupilumab or placebo and who had baseline and week 16 POEM assessments ICC Intraclass correlation coefficient, PGIC Patient Global Impression of Change, PGID Patient Global Impression of Disease, POEM Patient-Oriented Eczema Measure, $S D$ standard deviation, SEM standardized error of measurement

a Negative change scores denote improvement on POEM

b Computed as SD $\sqrt{1-r}$, where SD at baseline $=5.51$ and $r$ (test-retest ICC) $=0.64$ (baseline to week 2 for sample with no change on the PGID)

scales in adolescents (aged 12-17 years) with moderate-to-severe AD [24].

The results from the LIBERTY AD PEDS data indicate that a reasonable range of threshold estimates to define meaningful improvement in POEM scores would be -6.30 to -8.40 points, equating to a within-patient change in POEM score of 6-8 points. The lower bound of this range is in line with the mean estimate of 6.13 obtained by Howells et al. [33] using a patientreported (or parent-reported) global assessment (the P/PGA) anchor in a pediatric sample (children aged 1-15 years) with moderate-to-severe $\mathrm{AD}$. Therefore, based on the available evidence, 
Table 2 Estimates for thresholds for meaningful withinpatient change on the Children's Dermatology Life Quality Index using data from the phase 3 randomized, double-blind, placebo-controlled LIBERTY AD PEDS trial

\begin{tabular}{|c|c|c|}
\hline \multirow[t]{2}{*}{ Method } & \multicolumn{2}{|c|}{$\begin{array}{l}\text { Change in } \\
\text { CDLQI score } \\
\text { from baseline to } \\
\text { week } 16^{\mathrm{a}} \\
(N=356)\end{array}$} \\
\hline & Mean & Median \\
\hline \multicolumn{3}{|l|}{ Anchor based } \\
\hline $\begin{array}{l}\text { PGID improvement of } 1 \text { point } \\
(n=93)\end{array}$ & -7.30 & -6.00 \\
\hline $\begin{array}{l}\text { PGIC improvement "A little better" } \\
(n=85)\end{array}$ & -6.80 & -7.00 \\
\hline \multicolumn{3}{|l|}{ Distribution based } \\
\hline One-half SD at baseline & 3.69 & \\
\hline SEM $^{\mathrm{b}}$ & 3.52 & \\
\hline
\end{tabular}

The analysis sample included 356 randomized patients who received at least one dose of dupilumab or placebo and who had a baseline and week 16 CDLQI assessment CDLQI Children's Dermatology Life Quality Index

a Negative change scores denote improvement on CDLQI

b Computed as SD $\sqrt{1-r}$, where $\mathrm{SD}$ at baseline $=7.37$ and $r$ (test-retest ICC) $=0.77$ (baseline to week 2 for sample with no change on the PGID)

a minimum individual patient change score of 6 points in POEM total score is proposed as an appropriate threshold for defining a meaningful within-patient change.

The analyses for CDLQI indicate that a reasonable range of estimates to define meaningful improvement in CDLQI scores would be -6.80 to -7.30 points, equating to a within-patient change in CDLQI score of 6-7 points. One study has reported on the interpretation of scores on the CDLQI for children with psoriasis using data reported in the original validation study for the CDLQI [9] and considered that the equivalent one-half SD estimate for children with AD can be estimated as 2.8 [34]. Based on the available evidence and consistent with prior defined clinically meaningful within-patient change for the adolescent population [24], a minimum individual patient change score of 6 points in CDLQI total score is proposed as an appropriate threshold for defining a meaningful within-patient change.

Consistent with published studies [33, 35], POEM distribution estimates based on the trial data (2.76 and 3.32) were considerably lower than the anchor-based estimates. Similarly, CDLQI distribution-based estimates of 3.52 and 3.69 are comparable with the one-half SD of 2.8 based on published CDLQI scores in AD [9] and considerably lower than the anchor-based estimates derived from the LIBERTY AD PEDS data. These findings are in line with earlier literature reporting on lower thresholds for distributionbased methods compared with anchor-based methods [36]. This study primarily used anchorbased methods to define thresholds of meaningful within-patient change in line with guidance from the US Food and Drug Administration on patient-reported outcome measures [29].

A limitation of this analysis is that thresholds were derived using empirically driven data of $\mathrm{AD}$ patients aged 6-11 years and may not be appropriate for extrapolation to other age groups or conditions.

\section{CONCLUSION}

In children with severe $\mathrm{AD}$, a minimum individual within-patient change of 6 points in POEM and CDLQI scores is proposed as an appropriate threshold for defining a clinically meaningful within-patient change.

\section{ACKNOWLEDGEMENTS}

Funding. The study was sponsored by Sanofi and Regeneron Pharmaceuticals, Inc. The journal Rapid Service Fee was sponsored by Sanofi Genzyme and Regeneron Pharmaceuticals, Inc. All authors had full access to all of the data in this study and take complete responsibility for the integrity of the data and accuracy of the data analysis. 
Medical writing and editorial assistance. Medical writing and editorial assistance provided by Juliet Bell, PhD, and Liselotte van Delden, of Excerpta Medica, funded by Sanofi Genzyme and Regeneron Pharmaceuticals, Inc., according to the Good Publication Practice guideline.

Authorship. All named authors meet the International Committee of Medical Journal Editors (ICMJE) criteria for authorship for this article, take responsibility for the integrity of the work as a whole, and have given their approval for this version to be published.

Author contributions. All authors contributed to manuscript concept and design. ELS and MdB-W acquired data. ZC conducted the statistical analyses on the data. All authors interpreted the data, provided critical feedback on the manuscript, approved the final manuscript for submission, and are accountable for the accuracy and integrity of the manuscript. The authors acknowledge Dr. Abhijit Gadkari and Dr. Laurent Eckert for earlier contributions to the study design and data analysis.

Disclosures. Eric L. Simpson reports personal fees from AbbVie, Boehringer-Ingelheim, Collective Acument LLC, Eli Lilly, Forte Bio, Incyte, Janssen, Kyowa Hakko Kirin, Leo Pharma, Novartis, Ortho Dermatologics, Pfizer, Pierre Fabre Dermo Cosmetique, Regeneron, Roivant, Sanofi and Valeant; and reports grants (or Principal Investigator role) from AbbVie, Eli Lilly, Incyte, Kyowa Hakko Kirin, Leo Pharma, Merck, Novartis, Pfizer, Regeneron, Sanofi, Tioga, and Vanda. Marjolein de Bruin-Weller has been Principal Investigator, advisory board member, and consultant for Regeneron Pharmaceuticals, Inc., Sanofi Genzyme; Principal Investigator and advisory board member for AbbVie and Pfizer; and advisory board member for Eli Lilly and UCB. Ashish Bansal is an employee and shareholder of Regeneron Pharmaceuticals, Inc. Zhen Chen is an employee and shareholder of Regeneron Pharmaceuticals, Inc. Lauren Nelson and Diane Whalley are employees of RTI Health Solutions, which received funding from Regeneron
Pharmaceuticals, Inc. Randy Prescilla is an employee of Sanofi Genzyme, may hold stock and/or stock options in Sanofi. Isabelle Guillemin was an employee of Sanofi at the time of the research, may hold stock and/or stock options in Sanofi. Dimittri Delevry is an employee and shareholder of Regeneron Pharmaceuticals, Inc.

Compliance with ethics guidelines. The study was conducted in accordance with the provisions of the Declaration of Helsinki, the International Council for Harmonisation Good Clinical Practice (ICH GCP) guideline, and applicable regulatory requirements; the protocol was reviewed and approved by institutional review boards/ethics committees. For all patients, written informed consent was obtained from a parent or legal guardian, and written informed assent was obtained from the patient.

Data availability. Qualified researchers may request access to study documents (including the clinical study report, study protocol with any amendments, blank case report form, statistical analysis plan) that support the methods and findings reported in this manuscript. Individual anonymized participant data will be considered for sharing once the indication has been approved by a regulatory body, if there is legal authority to share the data and there is not a reasonable likelihood of participant re-identification. Requests should be submitted to https://vivli.org/.

Open Access. This article is licensed under a Creative Commons Attribution-NonCommercial 4.0 International License, which permits any non-commercial use, sharing, adaptation, distribution and reproduction in any medium or format, as long as you give appropriate credit to the original author(s) and the source, provide a link to the Creative Commons licence, and indicate if changes were made. The images or other third party material in this article are included in the article's Creative Commons licence, unless indicated otherwise in a credit line to the material. If material is not included in the article's Creative Commons licence and 
your intended use is not permitted by statutory regulation or exceeds the permitted use, you will need to obtain permission directly from the copyright holder. To view a copy of this licence, visit http://creativecommons.org/licenses/by$\mathrm{nc} / 4.0 /$.

\section{REFERENCES}

1. Bieber T. Atopic dermatitis. N Engl J Med. 2008;358: 1483-94.

2. Murray CJ, Richards MA, Newton JN, et al. UK health performance: findings of the Global Burden of Disease Study 2010. Lancet. 2013;381:997-1020.

3. Margolis JS, Abuabara K, Bilker W, Hoffstad O, Margolis DJ. Persistence of mild to moderate atopic dermatitis. JAMA Dermatol. 2014;150(6):593-600.

4. Brown SJ. Atopic eczema. Clin Med (Lond). 2016;16:66-9.

5. Deckers IA, McLean S, Linssen S, Mommers M, van Schayck CP, Sheikh A. Investigating international time trends in the incidence and prevalence of atopic eczema 1990-2010: a systematic review of epidemiological studies. PLoS ONE. 2012;7:e39803.

6. Illi S, von Mutius E, Lau S, et al. The natural course of atopic dermatitis from birth to age 7 years and the association with asthma. J Allergy Clin Immunol. 2004;113:925-31.

7. Charman CR, Venn AJ, Williams HC. The PatientOriented Eczema Measure: development and initial validation of a new tool for measuring atopic eczema severity from the patients' perspective. Arch Dermatol. 2004;140:1513-9.

8. Chalmers JR, Simpson E, Apfelbacher CJ, et al. Report from the fourth international consensus meeting to harmonize core outcome measures for atopic eczema/dermatitis clinical trials (HOME initiative). Br J Dermatol. 2016;175:69-79.

9. Lewis-Jones MS, Finlay AY. The Children's Dermatology Life Quality Index (CDLQI): initial validation and practical use. Br J Dermatol. 1995;132: 942-9.

10. Salek MS, Jung S, Brincat-Ruffini LA, et al. Clinical experience and psychometric properties of the Children's Dermatology Life Quality Index (CDLQI), 1995-2012. Br J Dermatol. 2013;169: 734-59.
11. Coutanceau C, Stalder JF. Analysis of correlations between patient-oriented SCORAD (PO-SCORAD) and other assessment scores of atopic dermatitis severity and quality of life. Dermatology. 2014;229: 248-55.

12. Armstrong AW, Johnson MA, Lin S, Maverakis E, Fazel N, Liu FT. Patient-centered, direct-access online care for management of atopic dermatitis: a randomized clinical trial. JAMA Dermatol. 2015;151:154-60.

13. Zhong H, Deng X, Song Z, et al. Immunological changes after ASIT in AD allergen-specific immunotherapy and their potential correlation with clinical response in patients with atopic dermatitis patients sensitized to house dust mite. J Eur Acad Dermatol Venereol. 2015;29:1318-24.

14. Drake L, Prendergast M, Maher R, et al. The impact of tacrolimus ointment on health-related quality of life of adult and pediatric patients with atopic dermatitis. J Am Acad Dermatol. 2001;44:S65-72.

15. Aziah MS, Rosnah T, Mardziah A, Norzila MZ. Childhood atopic dermatitis: a measurement of quality of life and family impact. Med J Malaysia. 2002;57:329-39.

16. Holm EA, Wulf HC, Stegmann H, Jemec GB. Life quality assessment among patients with atopic eczema. Br J Dermatol. 2006;154:719-25.

17. Gånemo A, Svensson A, Lindberg M, Wahlgren CF. Quality of life in Swedish children with eczema. Acta Derm Venereol. 2007;87:345-9.

18. Hon KL, Leung TF, Ng PC, et al. Efficacy and tolerability of a Chinese herbal medicine concoction for treatment of atopic dermatitis: a randomized, double-blind, placebo-controlled study. Br J Dermatol. 2007; 157:357-63.

19. Weber MB, de Fontes Neto PT, Prati C, et al. Improvement of pruritus and quality of life of children with atopic dermatitis and their families after joining support groups. J Eur Acad Dermatol Venereol. 2008;22:992-7.

20. Ramírez-Anaya M, Macías ME, Velázquez-González E. Validation of a Mexican Spanish version of the Children's Dermatology Life Quality Index. Pediatr Dermatol. 2010;27:143-7.

21. Maksimović N, Janković S, Marinković J, Sekulović LK, Zivković Z, Spirić VT. Health-related quality of life in patients with atopic dermatitis. J Dermatol. 2012;39:42-7.

22. Ražnatović Djurović M, Janković J, Tomić Spirić V, Janković S. Health-related quality of life in children 
with moderate to severe atopic dermatitis. Acta Dermatovenerol Croat. 2015;23:178-84.

23. Wang IJ, Wang JY. Children with atopic dermatitis show clinical improvement after lactobacillus exposure. Clin Exp Allergy. 2015;45:779-87.

24. Simpson EL, de Bruin-Weller M, Eckert L, et al. Responder threshold for Patient-Oriented Eczema Measure (POEM) and Children's Dermatology Life Quality Index (CDLQI) in adolescents with atopic dermatitis. Dermatol Ther (Heidelb). 2019;9: 799-805.

25. Schram ME, Spuls PI, Leeflang MM, et al. EASI, (objective) SCORAD and POEM for atopic eczema: responsiveness and minimal clinically important difference. Allergy. 2012;67:99-106.

26. Basra MK, Salek MS, Camilleri L, et al. Determining the minimal clinically important difference and responsiveness of the dermatology life quality index (DLQI): further data. Dermatology. 2015;230: 27-33.

27. Paller AS, Siegfried EC, Thaçi D, et al. Efficacy and safety of dupilumab with concomitant topical corticosteroids in children 6 to 11 years old with severe atopic dermatitis: a randomized, double=blinded, placebo-controlled phase 3 trial. J Am Acad Dermatol. 2020;83(5):1282-93. https://doi.org/10. 1016/j.jaad.2020.06.054 (Erratum in: J Am Acad Dermatol. 2021;84(1):230).

28. Coon CD, Cook KF. Moving from significance to real-world meaning: methods for interpreting change in clinical outcome assessment scores. Qual Life Res. 2018;27:33-40.

29. US Food and Drug Administration. Guidance for industry. Patient-reported outcome measures: use in medical product development to support labeling claims. 2009. https://www.fda.gov/ downloads/drugs/guidances/ucm193282.pdf. Accessed July 2020.

30. Fayers PM, Hays RD. Don't middle your MIDs: regression to the mean shrinks estimates of minimally important differences. Qual Life Res. 2014;23: $1-4$.

31. Hays RD, Farivar SS, Liu H. Approaches and recommendations for estimating minimally important differences for health-related quality of life measures. COPD. 2005;2:63-7.

32. Revicki D, Hays RD, Cella D, Sloan J. Recommended methods for determining responsiveness and minimally important differences for patient-reported outcomes. J Clin Epidemiol. 2008;61:102-9.

33. Howells L, Ratib S, Chalmers JR, Bradshaw L, Thomas KS, CLOTHES trial team. How should minimally important change scores for the PatientOriented Eczema Measure be interpreted? A validation using varied methods. $\mathrm{Br} \mathrm{J}$ Dermatol. 2018;178:1135-42.

34. Langley RG, Paller AS, Hebert AA, et al. Patient-reported outcomes in pediatric patients with psoriasis undergoing etanercept treatment: 12-week results from a phase III randomized controlled trial. J Am Acad Dermatol. 2011;64:64-70.

35. Gaunt DM, Metcalfe C, Ridd M. The Patient-Oriented Eczema Measure in young children: responsiveness and minimal clinically important difference. Allergy. 2016;71:1620-5.

36. Wright A, Hannon J, Hegedus EJ, Kavckak AE. Clinimetrics corner; a closer look at the minimal clinically important difference (MCID). J Man Manip Ther. 2012;20:160-6. 